\title{
Safety Profile of the 9-Valent HPV Vaccine: A Combined Analysis of 7 Phase III Clinical Trials
}

\author{
Edson D. Moreira Jr, MD, PhD, ${ }^{\text {a }}$ Stan L. Block, MD, ${ }^{\mathrm{b}}$ Daron Ferris, MD, ${ }^{\mathrm{c}}$ Anna R. Giuliano, PhD, ${ }^{\mathrm{d}}$ Ole-Erik Iversen, \\ MD, elmar A. Joura, MD, ${ }^{f}$ Pope Kosalaraksa, MD, ${ }^{\circ}$ Andrea Schilling, MD, ${ }^{\text {h }}$ Pierre Van Damme, MD, PhD, ${ }^{i}$ \\ Jacob Bornstein, MD, MPA, ${ }^{j}$ F. Xavier Bosch, MD, ${ }^{k}$ Sophie Pils, MD, Jack Cuzick, PhD,' Suzanne M. Garland, \\ MD, ${ }^{m}$ Warner Huh, MD, ${ }^{n}$ Susanne K. Kjaer, MD, ${ }^{\circ}$ Hong Qi, MD, MPH, ${ }^{p}$ Donna Hyatt, BA, ${ }^{p}$ Jason Martin, MS, ${ }^{p}$ \\ Erin Moeller, MPH, ${ }^{p}$ Michael Ritter, BA, ${ }^{p}$ Martine Baudin, MD, ${ }^{q}$ Alain Luxembourg, MD, PhD ${ }^{p}$
}

OBJectives: The overall safety profile of the 9-valent human papillomavirus (9vHPV) vaccine was evaluated across 7 Phase III studies, conducted in males and females (nonpregnant at entry), 9 to 26 years of age.

METHODS: Vaccination was administered as a 3-dose regimen at day 1, and months 2 and 6. More than 15000 subjects received $\geq 1$ dose of 9 vHPV vaccine. In 2 of the studies, $>7000$ control subjects received $\geq 1$ dose of quadrivalent HPV (qHPV) vaccine. Serious and nonserious adverse events (AEs) and new medical conditions were recorded throughout the study. Subjects testing positive for pregnancy at day 1 were not vaccinated; those who became pregnant after day 1 were discontinued from further vaccination until resolution of the pregnancy. Pregnancies detected after study start $(n=2950)$ were followed to outcome. RESULTS: The most common AEs ( $\geq 5 \%)$ experienced by $9 \mathrm{vHPV}$ vaccine recipients were injection-site AEs (pain, swelling, erythema) and vaccine-related systemic AEs (headache, pyrexia). Injection-site AEs were more common in 9vHPV vaccine than qHPV vaccine recipients; most were mild-to-moderate in intensity. Discontinuations and vaccine-related serious AEs were rare $(0.1 \%$ and $<0.1 \%$, respectively). Seven deaths were reported; none were considered vaccine related. The proportions of pregnancies with adverse outcome were within ranges reported in the general population.

concLusions: The 9vHPV vaccine was generally well tolerated in subjects aged 9 to 26 years with an AE profile similar to that of the qHPV vaccine; injection-site AEs were more common with $9 \mathrm{vHPV}$ vaccine. Its additional coverage and safety profile support widespread $9 v H P V$ vaccination.

\footnotetext{
${ }^{a}$ Associação Obras Sociais Irmã Dulce and Oswaldo Cruz Foundation, Brazilian Ministry of Health, Bahia, Brazil; ${ }^{b}$ Kentucky Pediatric/Adult Research, Inc, Bardstown, Kentucky; 'Department of Medicine, Medical College of Georgia, Augusta University, Augusta, Georgia; ${ }^{d}$ Center for Infection Research in Cancer, Moffitt Cancer Center, Tampa, Florida; ${ }^{e}$ Department of Gynaecology, University of Bergen, Bergen, Norway; ${ }^{f}$ Department of Obstetrics, Medical University of Vienna, Vienna, Austria; 'Department of Medicine, Khon Kaen University, Khon Kaen, Thailand; ' Departamento de Ginecología y Obstetricia Clínica Alemana, Facultad de Medicina Clínica AlemanaUniversidad Del Desarrollo, Santiago, Chile; 'Centre for the Evaluation of Vaccination, Vaccine \& Infectious Disease Institute, University of Antwerp, Antwerp, Belgium; 'Department of Obstetrics and Gynecology, Galilee Medical Center and Bar Ilan University Faculty of Medicine, Nahariya, Israel; ${ }^{C}$ Catalan Institute of Oncology/ IDIBELL, Barcelona, Spain; 'Wolfson Institute of Preventive Medicine, London, United Kingdom; 'mRoyal Women's Hospital, University of Melbourne and Murdoch Childrens Research Institute, Parkville, Australia; ${ }^{n}$ Division of Gynecologic Oncology, University of Alabama Birmingham, Birmingham, Alabama; 'Danish Cancer Society Research Center and Department of Gynecology, Rigshospitalet, University of Copenhagen, Copenhagen, Denmark; ${ }^{P}$ Merck \& Co., Inc., Kenilworth, New Jersey; and ${ }^{q}$ Sanofi Pasteur MSD, Lyon, France.
}

WHAT'S KNOWN ON THIS SUBJECT: 9-valent HPV vaccination prevents infection and disease associated with vaccine HPV types in 16- to 26-year-old women. Efficacy findings were extended to 9- to 15-yearold girls and boys by demonstrating noninferior immunogenicity compared with women.

WHAT THIS STUDY ADDS: The additional coverage provided by the 9 -valent versus the quadrivalent $\mathrm{HPV}$ vaccine and a favorable safety profile (generally comparable to the quadrivalent vaccine and similar across age groups and genders) demonstrated herein, support widespread vaccination programs.

To cite: Moreira ED, Block SL, Ferris D, et al. Safety Profile of the 9-Valent HPV Vaccine: A Combined Analysis of 7 Phase III Clinical Trials. Pediatrics. 2016;138(2):e20154387 
TABLE 1 Phase III Studies of the 9vHPV Vaccine Contributing to the Combined Safety Analysis

\begin{tabular}{|c|c|c|c|c|}
\hline Study & Key Objectives & Safety Population & Vaccination & $\begin{array}{l}\text { Duration of Safety } \\
\text { Follow-up }\end{array}$ \\
\hline 001 & Immunogenicity, efficacy vs qHPV & Women aged $16-26$ y $(N=14185)$ & $\begin{array}{r}9 \mathrm{vHPV}: N=7092,{ }^{\mathrm{a}} \\
\text { qHPV: } N=7093\end{array}$ & Up to $72 \mathrm{mo}^{\mathrm{b}}$ \\
\hline 002 & $\begin{array}{l}\text { Adult-to-adolescent } \\
\text { immunobridging }\end{array}$ & $\begin{array}{l}\text { Girls }(n=1933) \text { and boys }(n=666) \text { aged } 9-15 \mathrm{y} \text {; } \\
\quad \text { women aged } 16-26 \text { y }(n=467)\end{array}$ & $9 v H P V: N=3066$ & $\begin{array}{l}\text { Girls/boys: } 36 \mathrm{mo} \\
\quad \text { women: } 12 \mathrm{mo}\end{array}$ \\
\hline 003 & Women-to-men immunobridging & $\begin{array}{l}\text { Men }(n=1416) \text { and women }(n=1099) \text { aged } \\
16-26 \text { y }\end{array}$ & $9 v H P V: N=2515$ & $12 \mathrm{mo}$ \\
\hline 005 & $\begin{array}{l}\text { Concomitant use: Menactra/ } \\
\text { Adacel }\end{array}$ & Girls $(n=620)$ and boys $(n=617)$ aged $11-15 \mathrm{y}$ & $9 v H P V: N=1237$ & $7 \mathrm{mo}$ \\
\hline 006 & $\begin{array}{l}\text { Assessment in previous qHPV } \\
\text { vaccine recipients }\end{array}$ & $\begin{array}{l}\text { Girls aged } 12-15 \text { y }(n=120) \text {; women aged } \\
\quad 16-26 \text { y }(n=493)\end{array}$ & $9 \mathrm{vHPV}: N=613^{\mathrm{c}}$ & $7 \mathrm{mo}$ \\
\hline 007 & Concomitant use: Repevax & Girls $(n=526)$ and boys $(n=526)$ aged $11-15$ y & $9 \mathrm{vHPV}: N=1052$ & $7 \mathrm{mo}$ \\
\hline 009 & qHPV-to-9vHPV immunobridging & Girls aged $9-15$ y $(N=598)$ & $\begin{array}{l}9 v H P V: N=300 \\
\text { gHPV: } N=298\end{array}$ & $7 \mathrm{mo}$ \\
\hline
\end{tabular}

Study 001 (NCT00543543; protocol V503-001); ; Study 002 (NCT00943722; protocol V503-002)9; Study 003 (NCT01651949; protocol V503-003) 10; Study 005 (NCT00988884, protocol V503-005)11; Study 006 (NCT01047345; protocol V503-006) $)^{12}$; Study 007 (NCT01073293; protocol V503-007) ${ }^{13}$; Study 009 (NCT01304498; protocol V503-009/GDS01C). ${ }^{14} \mathrm{~N} / n=s u b j e c t s$ who received at least 1 vaccination and did not receive a mixed vaccine regimen. A total of 15875 subjects received at least 1 vaccination in these studies. Most subjects (97.2\%; 15 427 of 15875$)$ received the 3 vaccinations.

a Subjects who received the low-dose or high-dose formulation of 9vHPV vaccine during the dose selection portion of the study8,15 are not considered in this report; safety findings in these subjects are reported in Luxembourg et al. ${ }^{16}$

${ }^{b}$ Visit cutoff date: March 10, 2014; maximum follow-up 72 mo after vaccination dose 1 (median: 48 mo)

${ }^{\circ}$ Subjects who received placebo in Study 006 are not considered in this report; safety findings in these subjects are reported. ${ }^{12}$

A 9-valent HPV (9vHPV; types $6 / 11 / 16 / 18 / 31 / 33 / 45 / 52 / 58)$ vaccine (Gardasil 9, Merck \& Co, Inc, Kenilworth, NJ) was developed to provide protection against the HPV types already covered by the quadrivalent HPV (qHPV; types $6 / 11 / 16 / 18$ ) vaccine and the next 5 most common oncogenic types associated with cervical cancer worldwide (types 31/33/45/52/58). ${ }^{1}$ The 9vHPV vaccine could potentially prevent $\sim 90 \%$ of cervical cancers; HPV-related vulvar, vaginal, and anal cancers; and genital warts worldwide. ${ }^{2-6}$ The 9vHPV vaccine was licensed in 2014 in the United States and in 2015 in Canada, the European Union, and Australia. In 2015, the Advisory Committee on Immunization Practices recommended routine vaccination of girls and boys aged 11 and 12 years not previously vaccinated with any HPV vaccine. ${ }^{7}$ Safety and adverse events (AEs) were assessed in 7 Phase III studies in $>15000$ male and female subjects aged 9 to 26 years. ${ }^{8-14}$ This report summarizes the safety results across these 7 clinical trials.

\section{METHODS}

\section{Enrollment}

The studies included in this report are summarized in Table 1. Each study was conducted in accordance with principles of Good Clinical Practice and approved by the institutional review board at each participating institution and by regulatory agencies. Written informed consent was provided by all adult subjects and by a parent or legal guardian of subjects who were minors. An external Data Monitoring Committee assessed safety findings during the studies. Baseline characteristics for the overall population of subjects who received the 9vHPV vaccine are presented in Table 2.

\section{Vaccination}

\section{A 3-dose vaccination regimen given} intramuscularly at day 1 and months 2 and 6 was evaluated in each study. In studies 001 and 009 , subjects were randomized to receive 9vHPV or qHPV vaccines. In study 006 , subjects were randomized to receive
TABLE 2 Subjects Characteristics

\begin{tabular}{|c|c|}
\hline & 9vHPV Vaccine \\
\hline Subjects in population, $n$ & 15875 \\
\hline \multicolumn{2}{|l|}{ Sex, $n(\%)$} \\
\hline Male & 3225 (20.3) \\
\hline Female & $12650(79.7)$ \\
\hline \multicolumn{2}{|l|}{ Age, $n(\%)$} \\
\hline $9-15$ y & $5308(33.4)$ \\
\hline $16-17$ y & $608(3.8)$ \\
\hline $18-26$ y & 9959 (62.7) \\
\hline Mean (SD) & $18.4(5.1)$ \\
\hline Median (range) & $20.0(9-26)$ \\
\hline \multicolumn{2}{|l|}{ Race, $n(\%)$} \\
\hline Asian & 2213 (13.9) \\
\hline Black & $718(4.5)$ \\
\hline Othera & 3628 (22.9) \\
\hline White & $9316(58.7)$ \\
\hline \multicolumn{2}{|l|}{ Region ${ }^{b}$} \\
\hline Africa & $215(1.4)$ \\
\hline Asia-Pacific & $2112(13.3)$ \\
\hline Europe & $5660(35.7)$ \\
\hline Latin America & $4186(26.4)$ \\
\hline North America & $3702(23.3)$ \\
\hline \multicolumn{2}{|c|}{$\begin{array}{l}\text { a Mostly multiracial subjects. } \\
\text { b Study participants were from } 31 \text { countries (Australia, } \\
\text { Austria, Belgium, Brazil, Canada, Chile, Colombia, Costa } \\
\text { Rica, Denmark, Finland, Germany, Hong Kong, India, } \\
\text { Israel, Italy, Japan, Korea, Malaysia, Mexico, New Zealand, } \\
\text { Norway, Peru, the Philippines, Poland, South Africa, Spain, } \\
\text { Sweden, Taiwan, Thailand, Turkey, and the United States } \\
\text { [including Puerto Rico]). }\end{array}$} \\
\hline
\end{tabular}

9vHPV vaccine or saline placebo. In studies 002, 003, 005, and 007, all participants received $9 \mathrm{vHPV}$ vaccine. 


\section{Safety Evaluation}

Vaccination Report Card-Aided Surveillance

Subjects were observed for $\geq 30$ minutes after each vaccination for any immediate reaction, with particular attention to any evidence of a hypersensitivity reaction. All subjects received a Vaccination Report Card (VRC) at each vaccination visit. Subjects were asked to record their oral temperature on the VRC in the evening after each vaccination and daily thereafter for 4 days. Injectionsite and systemic AEs were recorded on the VRC for a total of 15 days including the day of vaccination. The VRC prompted the recording of injection-site AEs of pain, swelling, and erythema for 5 days including the day of vaccination. For each $\mathrm{AE}$, participants were asked to rate the symptom as mild (awareness of sign or symptom but easily tolerated), moderate (discomfort enough to cause interference with usual activities), or severe (incapacitating with inability to work or do usual activity); injection-site AEs of swelling and erythema were rated by size. Investigators were instructed to assign causality to AEs on the basis of exposure, time course, likely cause, and consistency with the vaccine's known profile. Vaccine-related AEs were those that were determined by the investigator to be possibly, probably, or definitely vaccine related.

\section{Serious AES}

Serious AEs were predefined as any $\mathrm{AE}$ that resulted in death, was deemed by the investigator to be life threatening or a persistent or significant disability or incapacity, resulted in or prolonged an existing in-patient hospitalization, or was a congenital anomaly, a cancer, or another important medical event. Deaths and serious vaccine-related AEs were collected for the entire study duration in all studies. Other serious AEs were collected from day 1 through 6 months after the last vaccination in studies that lasted $\geq 12$ months (studies 001, 002, 003), or from day 1 through study-end for studies that lasted 7 months (studies 005, 006, 007, 009). Fetal losses were reported as serious AEs for any pregnancy with a last menstrual period $<6$ months after the last vaccination (or before study end for studies of 7-month duration). Additionally, investigators could report any serious vaccine-related AEs occurring after subject study termination.

\section{New Medical Conditions}

New medical events occurring outside a day 1 to 15 postvaccination period and not reported as serious AEs were reported as new medical conditions. New medical conditions were collected at each scheduled visit for the entire duration of the studies; collection of new medical condition data were prespecified in the study protocols and was mandatory.

\section{Pregnancy-Related Events.}

All female subjects underwent pregnancy testing based on urine or serum analyses for $\beta$-human chorionic gonadotropin ( $\beta$-hCG) before each vaccination. Participants found to be pregnant at day 1 were not vaccinated. Females aged $\geq 16$ years at enrollment were instructed to use effective contraception through month 7. Participants who inadvertently became pregnant before receiving all 3 doses of vaccine did not receive additional doses until $\geq 4$ weeks after resolution of pregnancy and normalization of $\beta$-hCG levels. If pregnancy was detected after completion of the vaccine series, women completed the study visits and procedures per protocol at the investigator's discretion. Pregnancies occurring after day 1 were followed to outcome. Serious AEs for infants born to study participants were collected throughout the study and followed to outcome.

\section{Data Analysis}

All participants who received $\geq 1$ study vaccination and had follow-up data are included in the analysis of safety with the following exceptions: (1) subjects enrolled in the dose-selection portion of study 001 who received 9vHPV vaccine dose formulations not selected for Phase III evaluation, that is, lowdose and high-dose 9vHPV vaccine (previously reported) ${ }^{15,16}$; (2) subjects randomized to the saline placebo arm of study 006 (previously reported; $n=306)^{12}$; and (3) a small number of subjects $(<0.1 \%)$ who accidentally received noncompliant dosing regimens (eg, mixed regimens of 9vHPV and qHPV vaccine or mixed regimen of 9vHPV vaccine and placebo). This combined analysis of safety data provides a cross-study summary of AEs and new medical conditions, described as frequencies and percentages across study group and type of event.

\section{RESULTS}

\section{AEs, Serious AEs, and New Medical Conditions}

The incidences of AEs were generally similar in younger ( $9-15$ years) and older (16-26 years) female patients. In study 001 in female subjects 16 to 26 years of age, the most common injection-site AEs (incidence $\geq 5 \%$ ) were pain, swelling, and erythema (reported in 89.9\%, 40.0\%, and $34.0 \%$ of subjects after $9 \mathrm{vHPV}$, and 83.5\%, 28.8\%, and $25.6 \%$ after qHPV vaccinations, respectively, over entire series of injections; Table 3); these AEs were more frequent with $9 v H P V$ vaccine; the difference was statistically significant $(P<.001)$ for the 3 AEs. In study 009 in female subjects 9 to 15 years of age, the most common injection-site AEs (incidence $\geq 5 \%$ ) were also pain, swelling, and 
erythema (reported in $89.3 \%, 47.8 \%$, and $34.1 \%$ of subjects after $9 \mathrm{vHPV}$ and $88.3 \%, 36.0 \%$, and $29.3 \%$ of subjects after qHPV vaccinations respectively, over entire series of injections; Table 4). These AEs were more frequent with 9vHPV vaccine but was statistically significant $(P=.003)$ only for swelling. In both studies, most injection-site AEs were mild to moderate in intensity and increased with subsequent doses for both vaccines. Severe injectionsite AEs increased with subsequent doses (in study 001, severe injectionsite pain increased from $0.7 \%$ or $0.4 \%$ after dose 1 to $2.6 \%$ or $1.7 \%$ after dose 3 with 9vHPV and qHPV vaccines, respectively). The most common vaccine-related systemic AEs (incidence $\geq 5 \%$ ) for $9 \mathrm{vHPV}$ vaccine were headache and pyrexia (reported in study 001 in $14.6 \%$ and $5.0 \%$ of subjects for $9 \mathrm{vHPV}$ and $13.7 \%$ and $4.3 \%$ for qHPV, and in study 009 in $11.4 \%$ and $5.0 \%$ of subjects for $9 \mathrm{vHPV}$ and $11.3 \%$ and $2.7 \%$ for qHPV vaccines, respectively) and were within the ranges previously reported in the qHPV vaccine and placebo arms of the qHPV vaccine clinical program (eg, headache and pyrexia in $18.7 \%$ and $10.1 \%$ of subjects for qHPV vaccine, and $19.9 \%$ and $8.4 \%$ for placebo, respectively). ${ }^{17}$ The frequencies of systemic AEs generally decreased with subsequent doses for both vaccines.

The AE profile in all subjects who received 9vHPV vaccine was similar to that seen in studies 001 and 009 (Table 5). The AE profile following $9 v H P V$ vaccination was similar between genders; AE frequencies were generally lower in male than female subjects. The 9vHPV vaccine AE profile was similar between races (Supplemental Table 8). Among 9vHPV vaccine recipients (Table 5), 7 subjects $(<0.1 \%)$ experienced serious vaccine-related AEs, and 7 subjects died during the studies. None of the deaths were considered vaccine related. Twenty subjects $(0.1 \%)$ discontinued vaccination because of an AE. Specific information regarding serious vaccine-related AEs, deaths, and AEs resulting in discontinuation is provided in the supplement. A total of 356 subjects $(2.3 \%)$ who received $9 \mathrm{vHPV}$ vaccine reported serious AEs including 51 (0.3\%) who reported serious AEs from day 1 to 15 after any vaccination (Table 6). The most common serious AEs were elective abortions, spontaneous abortions, and appendicitis; other serious AEs were of low frequency and affected various system organ classes. Only 1 $\mathrm{AE}$ of anaphylaxis was reported due to a nonstudy medication.

In study 001, new medical conditions (incident medical conditions occurring outside of the period of day 1 to 15 postvaccination and not considered serious AEs) were generally comparable between subjects who received 9vHPV or qHPV vaccines; frequencies of new medical conditions for each system organ class were similar between the 2 vaccines (Supplemental Table 9). In study 001 and among all 9vHPV vaccine recipients, the most frequent new medical conditions (incidence $\geq 5 \%$ ) were infections (Supplemental Tables 9 and 10).

Exploratory analyses of events of potential interest were performed. AEs of syncope occurred in 36 of the 15776 9vHPV vaccine recipients $(0.2 \%)$, including 22 cases after dose 1, 11 after dose 2 , and 3 after dose 3. Of the 36 cases, 20 occurred on the same day as vaccination. Events occurred mostly in female subjects (94\% [34 of 36]), did not cause any discontinuation from vaccination or the study, and did not reoccur after a subsequent dose. Incident AEs and new medical conditions potentially indicative of an autoimmune disorder were identified using a prespecified list of terms (Supplemental Table 11). In study 001, the frequencies of these conditions were similar between subjects in the 9vHPV
(3.9\%) and qHPV (3.6\%) vaccine groups (Supplemental Table 12). The types of conditions were generally similar between the 2 groups. The most common were arthralgia (9vHPV: 1.8\%; qHPV: 1.7\%) and thyroid (9vHPV: 1.2\%; qHPV: 1.0\%) conditions. Other conditions were low frequency $(\leq 0.1 \%)$, diverse, and affected various system organ classes. Among all 9vHPV vaccine recipients, the most common events were also arthralgia (1.3\%) and thyroid diseases (0.6\%); all other events were diverse and of low frequency $(\leq 0.1 \%$; Supplemental Table 13). Two subjects in study 001 ( 1 in each vaccine cohort) were diagnosed with complex regional pain syndrome (CRPS); both cases were attributed to a previous injury. Two subjects who received 9vHPV vaccine were diagnosed with postural orthostatic tachycardia syndrome (POTS), although 1 subject did not have recurrent episodes even after subsequent vaccinations, and the other subject had no temporal association (occurred $>3$ years after vaccination).

\section{Pregnancy-Related Events}

Pregnancy outcomes (including outcomes among live births through the neonatal period, ie, first 6 weeks of life) are shown in Table 7 (study 001) and Supplemental Table 14 (all 9vHPV vaccine recipients). Most pregnancies among 9vHPV vaccine recipients (95.9\% [1482 of 1546]) occurred in study 001 , which enrolled most of the women of childbearing age and had the longest follow-up. Most pregnancies resulted in live births $(80.1 \%$ for $9 v H P V$ and $79.2 \%$ for qHPV in study 001) and were largely vaginal deliveries. Elective terminations (in study $001,9.6 \%$ and $8.7 \%$ of pregnancies in 9vHPV and qHPV groups, respectively) were due to personal decision except for 9 pregnancies in study 001 (6 in 9vHPV and 3 in qHPV groups), which were terminated because of a congenital 


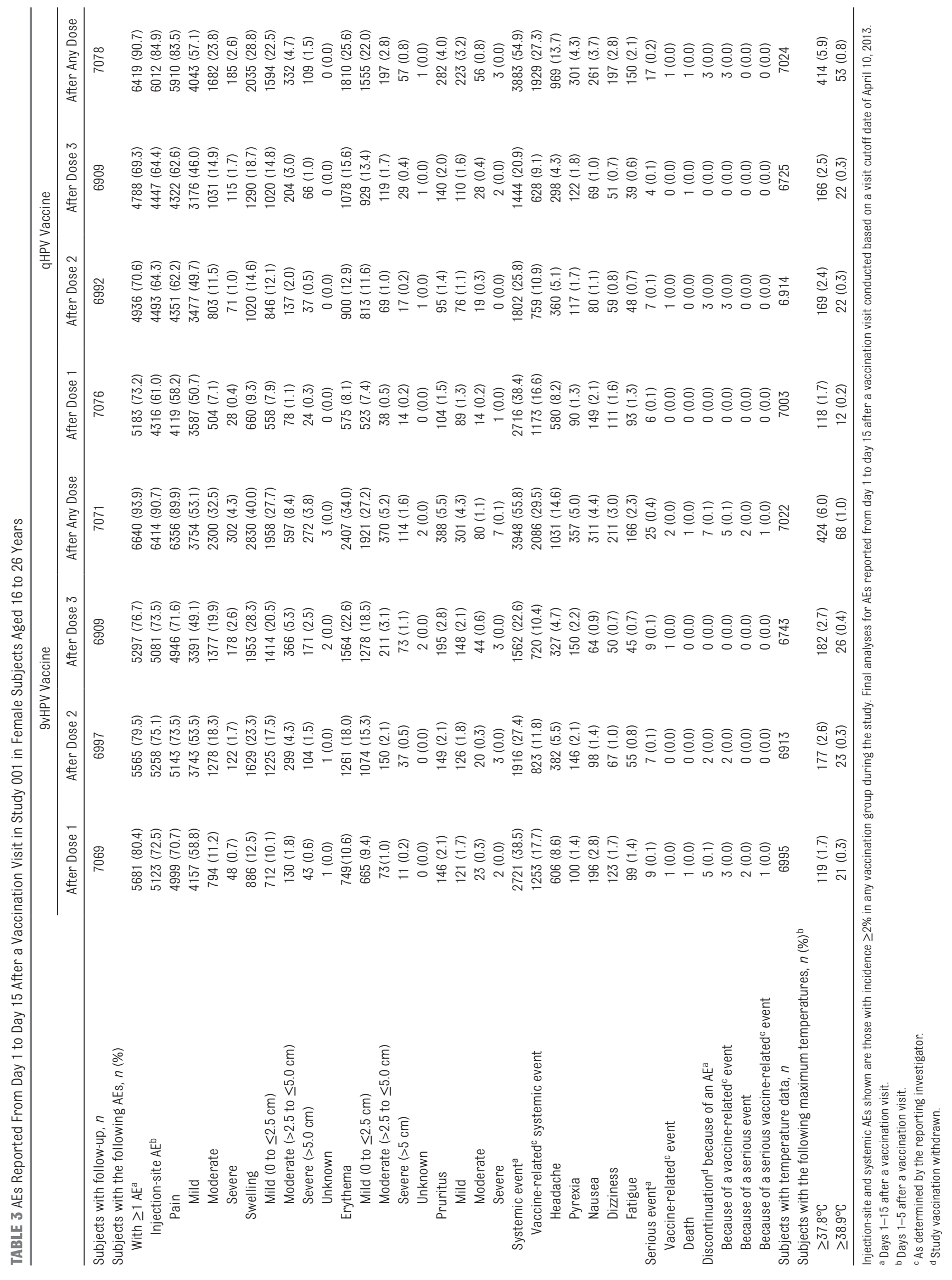




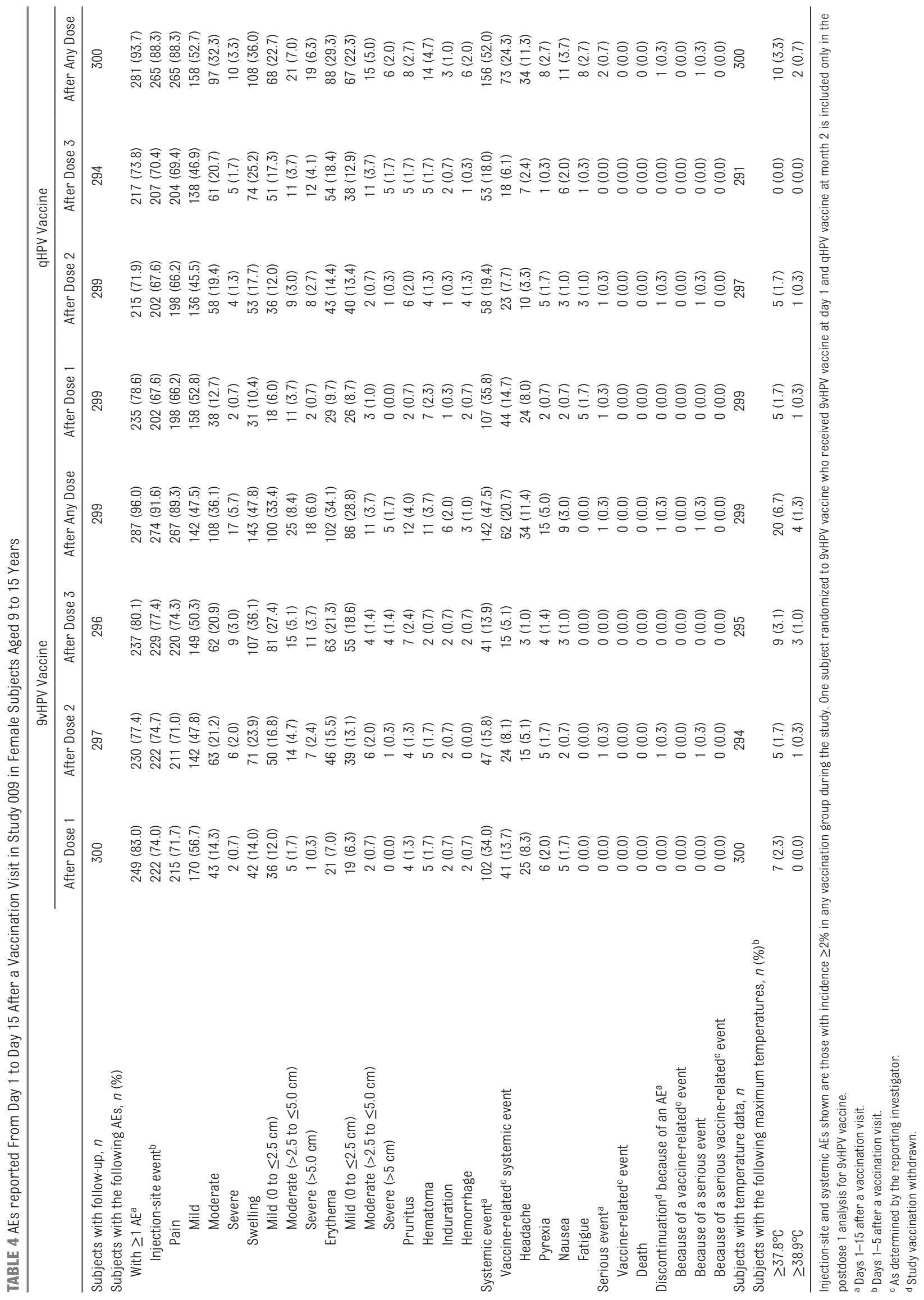


anomaly. No elective termination was due to a medical condition in the mother or a perceived risk due to vaccination. The rate of spontaneous abortions (number of spontaneous abortions per total number of pregnancy outcomes for which an outcome was known) in study 001 was $9.1 \%$ in the $9 v \mathrm{vHV}$ and $11.1 \%$ in the qHPV vaccine groups (rate was $9.2 \%$ among all subjects who received 9vHPV vaccine). Late fetal deaths (defined as fetal death taking place $>20$ weeks' gestation) occurred with a frequency of $<1 \%$ (relative to pregnancies with known outcome, excluding ectopic pregnancies and elective abortions).

Pregnancies with estimated dates of conception (EDC) within 30 days before or after any vaccination were considered to assess whether there could be an underlying high-risk period after conception. Because women were asked to use effective birth control only during the vaccination phase (day 1-month 7), only a small number of the EDC occurred within 30 days before or after a vaccination, representing in study $0015.8 \%$ (85 of 1459) and $6.1 \%$ (87 of 1435 ) in the 9vHPV and qHPV vaccine groups, respectively (Table 7) or $6.4 \%$ (97 of 1515) of all 9vHPV vaccine recipients (Supplemental Table 14), relative to the total number of pregnancies with known outcome. For pregnancies with EDC within 30 days before or

\begin{tabular}{|c|c|c|c|c|c|c|}
\hline & \multicolumn{6}{|c|}{ 9vHPV Vaccine } \\
\hline & \multicolumn{2}{|c|}{$\begin{array}{c}\text { All } \\
(N=15776)\end{array}$} & \multicolumn{2}{|c|}{$\begin{array}{c}\text { Female Subjects } \\
\quad(n=12583)\end{array}$} & \multicolumn{2}{|c|}{$\begin{array}{c}\text { Male Subjects } \\
\quad(n=3193)\end{array}$} \\
\hline & Count & $\%$ & Count & $\%$ & Count & $\%$ \\
\hline With $\geq 1 \mathrm{AEs}^{\mathrm{a}}$ & 14295 & 90.6 & 11660 & 92.7 & 2635 & 82.5 \\
\hline Injection-site event ${ }^{\mathrm{b}}$ & 13372 & 84.8 & 11085 & 88.1 & 2287 & 71.6 \\
\hline Pain & 13118 & 83.2 & 10937 & 86.9 & 2181 & 68.3 \\
\hline Mild & 8068 & 51.1 & 6480 & 51.5 & 1588 & 49.7 \\
\hline Moderate & 4497 & 28.5 & 3942 & 31.3 & 555 & 17.4 \\
\hline Severe & 552 & 3.5 & 514 & 4.1 & 38 & 1.2 \\
\hline Unknown & 1 & 0.0 & 1 & 0.0 & 0 & 0.0 \\
\hline Swelling & 5698 & 36.1 & 4918 & 39.1 & 780 & 24.4 \\
\hline Mild $(0$ to $\leq 2.5 \mathrm{~cm})$ & 3914 & 24.8 & 3348 & 26.6 & 566 & 17.7 \\
\hline Moderate $(>2.5$ to $\leq 5.0 \mathrm{~cm})$ & 1158 & 7.3 & 1036 & 8.2 & 122 & 3.8 \\
\hline Severe $(>5.0 \mathrm{~cm})$ & 618 & 3.9 & 526 & 4.2 & 92 & 2.9 \\
\hline Unknown & 8 & 0.1 & 8 & 0.1 & 0 & 0.0 \\
\hline Erythema & 4859 & 30.8 & 4145 & 32.9 & 714 & 22.4 \\
\hline Mild $(0$ to $\leq 2.5 \mathrm{~cm}$ ) & 3896 & 24.7 & 3304 & 26.3 & 592 & 18.5 \\
\hline Moderate $(>2.5$ to $\leq 5.0 \mathrm{~cm})$ & 698 & 4.4 & 611 & 4.9 & 87 & 2.7 \\
\hline Severe $(>5 \mathrm{~cm})$ & 251 & 1.6 & 216 & 1.7 & 35 & 1.1 \\
\hline Unknown & 14 & 0.1 & 14 & 0.1 & 0 & 0.0 \\
\hline Pruritus & 636 & 4.0 & 594 & 4.7 & 42 & 1.3 \\
\hline Mild & 487 & 3.1 & 454 & 3.6 & 33 & 1.0 \\
\hline Moderate & 138 & 0.9 & 130 & 1.0 & 8 & 0.3 \\
\hline Severe & 11 & 0.1 & 10 & 0.1 & 1 & 0.0 \\
\hline Systemic event ${ }^{c}$ & 8183 & 51.9 & 6772 & 53.8 & 1411 & 44.2 \\
\hline Vaccine-related ${ }^{\mathrm{d}}$ systemic event & 4217 & 26.7 & 3500 & 27.8 & 717 & 22.5 \\
\hline Headache & 2090 & 13.2 & 1765 & 14.0 & 325 & 10.2 \\
\hline Pyrexia & 955 & 6.1 & 734 & 5.8 & 221 & 6.9 \\
\hline Nausea & 503 & 3.2 & 451 & 3.6 & 52 & 1.6 \\
\hline Dizziness & 355 & 2.3 & 317 & 2.5 & 38 & 1.2 \\
\hline Fatigue & 294 & 1.9 & 249 & 2.0 & 45 & 1.4 \\
\hline Serious event ${ }^{\mathrm{a}}$ & 356 & 2.3 & 310 & 2.5 & 46 & 1.4 \\
\hline Vaccine-related ${ }^{d}$ event & 7 & 0.0 & 6 & 0.0 & 1 & 0.0 \\
\hline Death & 7 & 0.0 & 7 & 0.1 & 0 & 0.0 \\
\hline Discontinuation ${ }^{\mathrm{e}}$ because of an $\mathrm{AE}^{\mathrm{a}}$ & 20 & 0.1 & 15 & 0.1 & 5 & 0.2 \\
\hline Because of a vaccine-related ${ }^{d}$ event & 16 & 0.1 & 11 & 0.1 & 5 & 0.2 \\
\hline Because of a serious event & 5 & 0.0 & 4 & 0.0 & 1 & 0.0 \\
\hline Because of a serious vaccine-related ${ }^{\mathrm{d}}$ event & 2 & 0.0 & 1 & 0.0 & 1 & 0.0 \\
\hline
\end{tabular}

Injection-site and systemic AEs shown are those with incidence $\geq 2 \%$ in any vaccination group during the study. $N / n=$ Number of subjects as-treated who received at least 1 dose of the indicated vaccine and had at least 1 follow-up visit for an $\mathrm{AE}$.

${ }^{a}$ At any time during the study.

${ }^{b}$ Days 1 to 5 after any vaccination visit.

c Days 1 to 15 after any vaccination visit.

${ }^{\mathrm{d}}$ As determined by the reporting investigator.

e Study vaccination withdrawn 
after any vaccination, spontaneous abortion rates were $20.0 \%$ and $9.2 \%$ in study 001 , for subjects who received 9vHPV or qHPV vaccines, respectively, or $17.5 \%$ for all 9vHPV vaccine recipients. For pregnancies with known outcome with EDC not within 30 days before or after any vaccination, spontaneous abortion rates were $8.4 \%$ and $11.2 \%$ in study 001 for subjects who received 9vHPV or qHPV vaccines, respectively, or $8.6 \%$ for all $9 \mathrm{vHPV}$ vaccine recipients.

Reported congenital anomalies (Supplemental Table 15) including anomalies in live infants and fetal anomalies that resulted in elective abortions were diverse and affected various organs; none were considered vaccine related. No congenital anomaly was reported in pregnancies with EDC within 30 days before or after a vaccination with 9vHPV vaccine.

Forty-seven and 42 neonates born to women who received the 9vHPV vaccine and qHPV vaccine, respectively, experienced $\geq 1$ serious AE between birth and the first 6 weeks. None of these events were considered vaccine related. The most common events (excluding congenital anomalies) were infections (9vHPV: $n=11$; qHPV: $n=11$ ), prematurity (9vHPV: $n=8$; qHPV: $n=9$ ), and jaundice (9vHPV: $n=5$; qHPV: $n=2$ ).

\section{DISCUSSION}

In clinical trials, the 9vHPV vaccine was generally well tolerated and had an AE profile similar to that of the qHPV vaccine. Discontinuations due to an AE and serious vaccinerelated AEs were rare. Injectionsite AEs were more common with the 9vHPV than the qHPV vaccine, increased with subsequent doses for both vaccines, and were mostly mild-to-moderate in intensity. Frequencies of vaccine-related systemic AEs were generally similar between the 9vHPV and qHPV vaccine groups and within ranges previously reported in placebo recipients in the qHPV vaccine

TABLE 6 Subjects With Serious AEs During the Study, ${ }^{a}$ by System Organ Class, in Subjects Who Received 9vHPV Vaccine

\begin{tabular}{|c|c|c|c|c|}
\hline & \multicolumn{2}{|c|}{$\begin{array}{l}\text { Within } 15 \text { Days After Any Vaccination } \\
\qquad(n=15776)\end{array}$} & \multicolumn{2}{|c|}{$\begin{array}{l}\text { At Any Time } \\
(n=15778)\end{array}$} \\
\hline & Count & $\%$ & Count & $\%$ \\
\hline With $\geq 1$ serious AEs & 51 & 0.3 & 356 & 2.3 \\
\hline Blood and lymphatic system disorders & 2 & 0.0 & 2 & 0.0 \\
\hline Cardiac disorders & 0 & 0.0 & 3 & 0.0 \\
\hline Congenital, familial and genetic disorders & 0 & 0.0 & 1 & 0.0 \\
\hline Ear and labyrinth disorders & 1 & 0.0 & 1 & 0.0 \\
\hline Gastrointestinal disorders & 2 & 0.0 & 15 & 0.1 \\
\hline General disorders and administration site conditions & 2 & 0.0 & 6 & 0.0 \\
\hline Hepatobiliary disorders & 2 & 0.0 & 5 & 0.0 \\
\hline Immune system disorders & 3 & 0.0 & 4 & 0.0 \\
\hline Allergy to vaccine & 1 & 0.0 & 1 & 0.0 \\
\hline Anaphylactic reaction ${ }^{b}$ & 1 & 0.0 & 1 & 0.0 \\
\hline Hypersensitivity ${ }^{\mathrm{b}}$ & 1 & 0.0 & 1 & 0.0 \\
\hline Sarcoidosis & 0 & 0.0 & 1 & 0.0 \\
\hline Infections and infestations & 15 & 0.1 & 75 & 0.5 \\
\hline Injury, poisoning, and procedural complications & 7 & 0.0 & 25 & 0.2 \\
\hline Metabolism and nutrition disorders & 1 & 0.0 & 1 & 0.0 \\
\hline Musculoskeletal and connective tissue disorders & 0 & 0.0 & 4 & 0.0 \\
\hline Neoplasms benign, malignant and unspecified (including cysts and polyps) & 2 & 0.0 & 10 & 0.1 \\
\hline Nervous system disorders & 3 & 0.0 & 21 & 0.1 \\
\hline Pregnancy, puerperium and perinatal conditions & 1 & 0.0 & 70 & 0.4 \\
\hline Psychiatric disorders & 4 & 0.0 & 14 & 0.1 \\
\hline Renal and urinary disorders & 0 & 0.0 & 7 & 0.0 \\
\hline Reproductive system and breast disorders & 4 & 0.0 & 13 & 0.1 \\
\hline Respiratory, thoracic, and mediastinal disorders & 3 & 0.0 & 8 & 0.1 \\
\hline Skin and subcutaneous tissue disorders & 0 & 0.0 & 1 & 0.0 \\
\hline Surgical and medical procedures & 1 & 0.0 & 83 & 0.5 \\
\hline Vascular disorders & 1 & 0.0 & 5 & 0.0 \\
\hline
\end{tabular}

The summaries provided are subject count and percents calculated relative to the number of subjects as-treated. System organ class categories reported are those with incidence $>0 \%$ during the study. A subject is counted once within a category and may be counted in $>1$ category. $n=$ Number of subjects as-treated who received at least 1 dose of the indicated vaccine and had at least 1 follow-up visit for adverse event.

a Serious AEs were predefined as any AE that resulted in death, were deemed by the investigator to be life threatening, resulted in a persistent or significant disability or incapacity, resulted in or prolonged an existing in-patient hospitalization, or was a congenital anomaly, a cancer, or "other important medical event." Per protocol, serious AEs were reportable regardless of causality from day 1 through 180 days after the last vaccination (or for the entire study period for studies of 7 -month duration); events of fetal loss were to be reported as serious AEs for any pregnancy with a last menstrual period before 180 days after the last vaccination (or at any time during the study for studies of 7 -month duration); and deaths, serious vaccine-related AEs, and (in study 001) serious procedure-related AEs were to be reported for the entire duration of the study.

${ }^{\mathrm{b}}$ Allergic reaction to a nonstudy medication (anaphylactic reaction due to parenteral iron given for anemia, at 5 days after dose 3 ; hypersensitivity due to tramadol given for back pain, at 4 days after dose 3 ) 


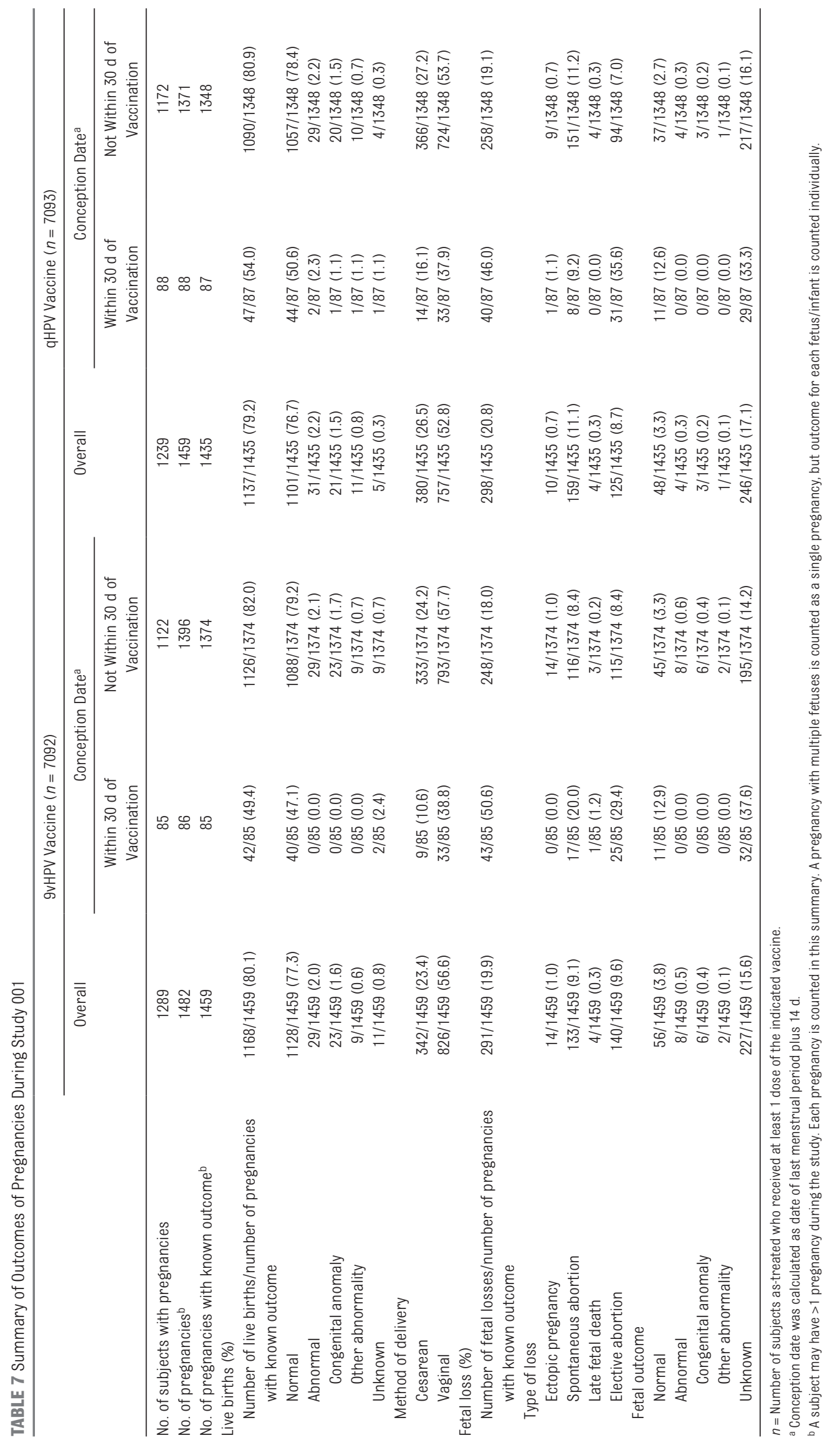


program. ${ }^{17}$ AEs were generally similar in nature and frequency between younger ( $9-15$ years) and older (16-26 years) female subjects and generally less common in male than female subjects. Serious AEs affected diverse system organ classes and did not indicate a specific safety concern. Events of syncope were rare, did not recur, and were not associated with serious sequelae. No anaphylactic reactions due to the vaccine were reported. The proportions of subjects with new medical conditions were similar in the 9vHPV and qHPV vaccine groups from study 001. Frequencies and types of incident conditions potentially indicative of an autoimmune disorder were similar between the 9vHPV and qHPV vaccines and also similar to those previously reported after qHPV vaccine or placebo administration. ${ }^{18}$ Study results did not suggest an association between 9vHPV vaccination and CRPS or POTS; of note, an assessment of the occurrence of CRPS and POTS in HPV vaccine recipients conducted by the European Medicines Agency produced similar conclusions. ${ }^{19}$

Women of childbearing age represented a large proportion of study participants. Therefore, all pregnancy outcomes were thoroughly documented. Pregnancy outcomes were generally similar among 9vHPV and qHPV vaccine recipients. For pregnancies with onset within 30 days before or after vaccination, spontaneous abortion rates were higher for the 9vHPV than qHPV vaccine; however, the frequencies of spontaneous abortions were within the ranges reported for pregnant women (ie, $\leq 33 \%$ for pregnancies detected with $\beta$-hCG), ${ }^{20-23}$ as well as ranges previously reported in similar analyses in the qHPV vaccine and placebo groups in the qHPV vaccine clinical program (see the online Supplemental Information for additional information). ${ }^{24-26}$

Congenital anomaly rates $(<2 \%$ of live births) were consistent with prevalences reported in the literature (ie, $3 \%-4 \%$ of all live births) and in the qHPV vaccine and placebo arms of the qHPV vaccine program. ${ }^{25-28}$ There was no clear pattern of anomaly types that differed from those occurring in pregnancies in the general population of the same age. All the congenital anomalies in the 9vHPV vaccine group occurred in pregnancies with onset $>30$ days after vaccination; thus, no highrisk period after conception was identified. The number and types of serious AEs that occurred among neonates were similar between the $9 v H P V$ and $\mathrm{qHPV}$ vaccines and none were considered vaccine related by the reporting investigator.

This combined analysis has several limitations. The overall safety database for 9vHPV vaccine is similar in size to that of the prelicensure database of qHPV vaccine ${ }^{18}$; however, it is insufficiently sized to identify AEs occurring at a rate $<1: 5200$. Such events are expected to be assessed in pharmacovigilance and postlicensure safety analyses. Because existing HPV vaccines prevent precancers due to HPV 16 and 18, the use of a placebo in subjects not previously vaccinated was deemed unethical. ${ }^{8,29}$ The qHPV vaccine was used as a control in 2 of the studies. Extensive clinical trial and postlicensure studies have reinforced the favorable safety profile of the qHPV vaccine in both sexes. ${ }^{18,30-40}$ The similarity between the safety profile of the $9 \mathrm{vHPV}$ vaccine and that of the $\mathrm{qHPV}$ vaccine supports the conclusion that the $9 \mathrm{vHPV}$ vaccine is generally well tolerated. The 9vHPV vaccine clinical program was not designed to provide a systematic assessment of the vaccine in pregnant women. Thus, a pregnancy registry for the $9 \mathrm{vHPV}$ vaccine has been established in the United States, based on the same process previously established for the pregnancy registry of the qHPV vaccine, ${ }^{41,42}$ to better describe the safety profile of pregnancy exposures to the $9 \mathrm{vHPV}$ vaccine.

\section{CONCLUSIONS}

In clinical trials, the 9vHPV vaccine was generally well tolerated in subjects aged 9 to 26 years. Its $\mathrm{AE}$ profile was similar to that of the qHPV vaccine; injection-site AEs were more common with the 9vHPV vaccine and mostly mild to moderate in intensity. The demonstrated efficacy and favorable safety profile of the 9vHPV vaccine support widespread vaccination programs.

\section{ACKNOWLEDGMENTS}

Thanks to Christine Durkan, Joy Ginanni, and Monica Rojas Meza of Merck \& Co, Inc for their outstanding work with documenting all the details of the serious adverse events and pregnancy-related events, and Scott Vuocolo, PhD, Joanne E. Tomassini, PhD, Sheila Erespe, MS, and Carol Zecca, BS of Merck \& Co, Inc for assistance in the preparation and submission of the manuscript.

\section{ABBREVIATIONS}

AEs: adverse events

$\beta$-hCG: $\beta$-human chorionic gonadotropin

CRPS: complex regional pain syndrome

EDC: estimated dates of conception

HPV: human papillomavirus

9vHPV: 9-valent human papillomavirus

POTS: postural orthostatic tachycardia syndrome

qHPV: quadrivalent human papillomavirus

VRC: vaccination report card 
Dr Moreira conceptualized or designed the study, contributed to the acquisition of the data, interpreted the results, drafted the initial manuscript, and critically reviewed and/or revised the manuscript; Dr Block conceptualized or designed the study, contributed to the analysis and acquisition of the data, interpreted the results, drafted the initial manuscript, and reviewed and/or revised the manuscript; Dr Ferris contributed to acquisition of the data and reviewed and/or revised the manuscript; Dr Giuliano contributed to the interpretation of the results and reviewed and/or revised the manuscript; Drs Iversen, Joura, and Kosalaraksa contributed to the acquisition of the data, interpreted the results, and reviewed and/or revised the manuscript; Dr Schilling contributed to the analysis and acquisition of the data, interpreted the results, drafted the initial manuscript, and reviewed and/or revised the manuscript; Dr Van Damme contributed to the acquisition of the data, interpreted results, and reviewed and/or revised the manuscript; Dr Bornstein contributed to the analysis and acquisition of the data, interpreted the results, and reviewed and/or revised the manuscript; Dr Bosch contributed to the interpretation of the results and reviewed and/or revised the manuscript; Dr Pils contributed to the acquisition of the data and reviewed and/or revised the manuscript; Dr Cuzick contributed to the analysis of the data, interpreted the results and reviewed and/or revised the manuscript; Dr Garland contributed to the interpretation of the results and reviewed and/or revised the manuscript; Dr Huh contributed to the analysis of the data and reviewed and/or revised the manuscript; Dr Kjaer contributed to the acquisition of the data, interpreted the results, and reviewed and/or revised the manuscript; $\mathrm{Dr}$ Qi contributed to the analysis of the data and reviewed and/or revised the manuscript; Ms Hyatt conceptualized or designed the study, contributed to the analysis and acquisition of the data, and reviewed and/or revised the manuscript; Mr Martin conceptualized or designed the study, contributed to the analysis and acquisition of the data, interpreted the results, drafted the initial manuscript, and reviewed and/or revised the manuscript; Ms Moeller conceptualized or designed the study, contributed to interpretation of the results, and reviewed and/or revised the manuscript; Mr Ritter substantially contributed to the interpretation of the results and reviewed and/or revised the manuscript; Dr Baudin conceptualized or designed the study, contributed to the analysis of the data, interpreted the results, and reviewed and/or revised the manuscript; Dr Luxembourg conceptualized or designed the study, contributed to the analysis of the data, interpretation of the results, drafted the initial manuscript, and critically reviewed and/or revised the manuscript; and all authors approved the final manuscript as submitted.

This trial has been registered at www.clinicaltrials.gov (identifiers NCT00543543, NCT00943722, NCT01651949, NCT00988884, NCT01047345, NCT01073293, and NCT01304498).

\section{DOI: $10.1542 /$ peds.2015-4387}

Accepted for publication May 12, 2016

Address correspondence to Edson D. Moreira Jr, MD, PhD, Centro de Pesquisa Gonçalo Moniz, Fundação 0swaldo Cruz, Rua Waldemar Falcão 121, Salvador, Bahia, Brasil, 40.296-710. E-mail: edson@bahia.fiocruz.br

PEDIATRICS (ISSN Numbers: Print, 0031-4005; Online, 1098-4275).

Copyright (๑) 2016 by the American Academy of Pediatrics

FINANCIAL DISCLOSURE: Dr Moreira reports grants and personal fees from Merck \& Co, Inc. Dr Block has received research grants from and is a member of a speaker's bureau for Merck \& Co, Inc. Dr Ferris has received research grants, financial compensation for consultation and advisory board work with Merck, and his institution has received financial support for other HPV vaccine-related studies from Merck. Dr Giuliano reports having received grant support and advisory board member fees to her institution from Merck. Dr Iversen reports having received compensation from Merck and GlaxoSmithKline to conduct vaccine clinical trials. Dr Joura reports having received grant support paid to his institution from Merck and GlaxoSmithKline and advisory board fees from Merck and Sanofi Pasteur MSD. Dr Kosalaraksa reports receiving past grant support from Merck through his institution. Dr Schilling has received research support for other HPV vaccine-related studies from Merck and honoraria outside the submitted work from Merck-Chile. Dr Van Damme acts as chief and principal investigator for vaccine trials conducted on behalf of the University of Antwerp, for which the university obtains research grants from vaccine manufacturers GlaxoSmithKline, Merck, and Sanofi Pasteur; speakers fees for presentations on vaccines are paid directly to an educational fund held by the University of Antwerp; he receives no personal remuneration for this work. Dr Bornstein reports grants from Merck Sharp \& Dohme, which are outside the submitted work. Dr Bosch reports having received institutional research and educational grants from Sanofi Pasteur MSD and GlaxoSmithKline and personal travel grant and speakers honorarium from Sanofi Pasteur MSD and GlaxoSmithKline. Dr Pils received travel support from Sanofi Pasteur. Dr Cuzick reports having received advisory board fees from Merck and GlaxoSmith Kline. Dr Garland reports having received grant support paid to her institution from GlaxoSmithKline, Merck, and CSL Bio and speakers honoraria for work performed in own time from Sanofi Pasteur and Merck. Warner Huh reports having received honoraria for advisory board participation with Merck. Dr Kjaer has received advisory board, speaker's fees, and unrestricted research grants through her institution from Merck. Dr Baudin is an employee of Sanofi Pasteur MSD. Ms Moeller, Mr Ritter, Dr Qi, Mr Martin, Ms Hyatt, and Dr Luxembourg are employees of Merck Sharp \& Dohme Corp, a subsidiary of Merck \& Co, Inc, Kenilworth, $\mathrm{NJ}$, and may own stock and/or stock options in the company.

FUNDING: Supported by Merck \& Co., Inc., Kenilworth, New Jersey.

POTENTIAL CONFLICT OF INTEREST: Dr Moreira reports grants and personal fees from Merck \& Co, Inc. Dr Block has received research grants from and is a member of a speaker's bureau for Merck \& Co, Inc. Dr Ferris has received research grants and financial compensation for consultation and advisory board work with Merck, and his institution has received financial support for other HPV vaccine-related studies from Merck. Dr Giuliano reports having received grant support and advisory board member fees to her institution from Merck. Dr Iversen reports having received compensation from Merck and GlaxoSmithKline to conduct vaccine clinical trials. Dr Joura reports having received grant support paid to his institution from Merck and GlaxoSmithKline; advisory board fees from Merck and Sanofi Pasteur MSD. Dr Kosalaraksa reports no conflict of interest. Dr Schilling has received research support for other HPV vaccine-related studies from Merck and honoraria outside the submitted work from Merck-Chile. Dr Van Damme reports research grants from vaccine manufacturers GlaxoSmithKline, Merck, and Sanofi Pasteur. Dr Bornstein reports grants from Merck Sharp \& Dohme outside the submitted work. Dr Bosch reports having received institutional research and educational grants from Sanofi Pasteur MSD and GlaxoSmithKline and personal travel grant and speakers honorarium from Sanofi Pasteur MSD and GlaxoSmithKline. Dr Pils reports travel support from Sanofi Pasteur. Dr Cuzick reports having received advisory board fees from Merck and GlaxoSmith Kline. Dr Garland reports having received grant support paid to her institution from GlaxoSmithKline, Merck, and CSL Bio and speakers honoraria for work performed on her own time from Sanofi Pasteur and Merck. Dr Huh reports having received honoraria for advisory board participation with Merck. Dr Kjaer received advisory board, speaker's fees, and unrestricted research grants through her institution from Merck. Dr Baudin is an employee of Sanofi Pasteur MSD. Ms Moeller, Mr Ritter, Dr Qi, Mr Martin, Ms Hyatt, and Dr Luxembourg are employees of Merck Sharp \& Dohme Corp, a subsidiary of Merck \& Co, Inc, Kenilworth, NJ, and may own stock and/or stock options in the company. 


\section{REFERENCES}

1. de Sanjose S, Quint WG, Alemany L, et al; Retrospective International Survey and HPV Time Trends Study Group. Human papillomavirus genotype attribution in invasive cervical cancer: a retrospective crosssectional worldwide study. Lancet Oncol. 2010;11(11):1048-1056

2. Serrano B, Alemany L, Tous S, et al. Potential impact of a nine-valent vaccine in human papillomavirus related cervical disease. Infect Agent Cancer. 2012;7(1):38

3. de Sanjosé S, Alemany L, Ordi J, et al; HPV VVAP study group. Worldwide human papillomavirus genotype attribution in over 2000 cases of intraepithelial and invasive lesions of the vulva. Eur $J$ Cancer. 2013:49(16):3450-3461

4. Alemany L, Saunier M, AlvaradoCabrero I, et al; HPV VVAP Study Group. Human papillomavirus DNA prevalence and type distribution in anal carcinomas worldwide. Int J Cancer. 2015;136(1):98-107

5. Alemany L, Saunier M, Tinoco L, et al; HPV VVAP study group. Large contribution of human papillomavirus in vaginal neoplastic lesions: a worldwide study in 597 samples. Eur J Cancer. 2014;50(16):2846-2854

6. Lacey CJ, Lowndes CM, Shah KV. Chapter 4: Burden and management of non-cancerous HPV-related conditions: HPV-6/11 disease. Vaccine. 2006;24(suppl 3):S3, 35-41

7. Petrosky E, Bocchini JA Jr, Hariri S, et al; Centers for Disease Control and Prevention (CDC). Use of 9-valent human papillomavirus (HPV) vaccine: updated HPV vaccination recommendations of the advisory committee on immunization practices. MMWR Morb Mortal Wkly Rep. 2015;64(11):300-304

8. Joura $E A$, Giuliano AR, Iversen $0 E$, et al; Broad Spectrum HPV Vaccine Study. A 9-valent HPV vaccine against infection and intraepithelial neoplasia in women. $\mathrm{N} \mathrm{Engl} \mathrm{J} \mathrm{Med.}$ 2015;372(8):711-723

9. Van Damme P, Olsson SE, Block S, et al. Immunogenicity and safety of a 9-valent HPV vaccine. Pediatrics. 2015;136(1). Available at: www. pediatrics.org/cgi/content/full/136/1/ e28

10. Castellsagué X, Giuliano AR, Goldstone $S$, et al. Immunogenicity and safety of the 9-valent HPV vaccine in men. Vaccine. 2015;33(48):6892-6901

11. Schilling A, Parra MM, Gutierrez M, et al. Coadministration of a 9-valent human papillomavirus vaccine with meningococcal and Tdap vaccines. Pediatrics. 2015;136(3). Available at: www.pediatrics.org/cgi/content/full/ 136/3/e563

12. Garland SM, Cheung TH, McNeill S, et al. Safety and immunogenicity of a 9-valent HPV vaccine in females 12-26 years of age who previously received the quadrivalent HPV vaccine. Vaccine. 2015;33(48):6855-6864

13. Kosalaraksa P, Mehlsen J, Vesikari T, et al. An open-label, randomized study of a 9-valent human papillomavirus vaccine given concomitantly with diphtheria, tetanus, pertussis and poliomyelitis vaccines to healthy adolescents 11-15 years of age. Pediatr Infect Dis J. 2015;34(6):627-634

14. Vesikari T, Brodszki N, van Damme $P$, et al. A randomized, double-blind, phase iii study of the immunogenicity and safety of a 9-valent human papillomavirus I1 virus-like particle vaccine (V503) versus Gardasil ${ }^{\circledR}$ in 9-15-year-old girls. Pediatr Infect Dis J. 2015;34 (9):992-998

15. Chen $Y H$, Gesser R, Luxembourg $A$. A seamless phase IIB/III adaptive outcome trial: design rationale and implementation challenges. Clin Trials. 2015;12(1):84-90

16. Luxembourg A, Brown D, Bouchard C, et al. Phase II studies to select the formulation of a multivalent HPV L1 virus-like particle (VLP) vaccine. Hum Vaccin Immunother. 2015;11(6):1313-1322

17. GARDASIL. EPAR - Scientific discussion 05/10/2006. Available at: http:// www.ema.europa.eu/docs/en_ GB/document_library/EPAR_-_ Scientific_Discussion/human/000703/ WC500021140.pdf. Accessed March 21, 2016
18. Block SL, Brown DR, Chatterjee A, et al. Clinical trial and post-licensure safety profile of a prophylactic human papillomavirus (types 6, 11, 16, and 18) 11 virus-like particle vaccine. Pediatr Infect Dis J. 2010;29(2):95-101

19. European Medicines Agency. Review concludes evidence does not support that HPV vaccines cause CRPS or POTS. 2015. Available at: http://www. ema.europa.eu/ema/index.jsp?curl= pages/news_and_events/news/2015/ 11/news_detail_002429.jsp\&mid= WC0b01ac058004d5c1. Accessed November 29, 2015

20. Wilcox AJ, Weinberg CR, O'Connor JF, et al. Incidence of early loss of pregnancy. N Engl J Med. 1988;319(4):189-194

21. Wilcox AJ, Baird DD, Weinberg CR. Time of implantation of the conceptus and loss of pregnancy. N Engl J Med. 1999;340(23):1796-1799

22. Lohstroh PN, Overstreet JW, Stewart $\mathrm{DR}$, et al. Secretion and excretion of human chorionic gonadotropin during early pregnancy. Fertil Steril. 2005;83(4):1000-1011

23. García-Enguídanos A, Calle ME, Valero J, Luna S, Domínguez-Rojas V. Risk factors in miscarriage: a review. Eur J Obstet Gynecol Reprod Biol. 2002;102(2):111-119

24. Garland SM, Hernandez-Avila M, Wheeler CM, et al; Females United to Unilaterally Reduce Endo/Ectocervical Disease (FUTURE) I Investigators. Quadrivalent vaccine against human papillomavirus to prevent anogenital diseases. N Engl J Med. 2007;356(19):1928-1943

25. FUTURE II Study Group. Quadrivalent vaccine against human papillomavirus to prevent high-grade cervical lesions. N Engl J Med. 2007;356(19) 1915-1927

26. Garland SM, Ault KA, Gall SA, et al; Quadrivalent Human Papillomavirus Vaccine Phase III Investigators. Pregnancy and infant outcomes in the clinical trials of a human papillomavirus type 6/11/16/18 vaccine: a combined analysis of five randomized controlled trials. Obstet Gynecol. 2009;114(6):1179-1188 
27. Hobbs CA, Cleves MA, Simmons CJ. Genetic epidemiology and congenital malformations: from the chromosome to the crib. Arch Pediatr Adolesc Med. 2002;156(4):315-320

28. Hollier LM, Leveno KJ, Kelly MA, MCIntire DD, Cunningham FG. Maternal age and malformations in singleton births. Obstet Gynecol. 2000;96 (5 Pt 1):701-706

29. Luxembourg A, Bautista 0, Moeller E, Ritter M, Chen J. Design of a large outcome trial for a multivalent human papillomavirus L1 virus-like particle vaccine. Contemp Clin Trials. 2015;42:18-25

30. Moreira ED Jr, Palefsky JM, Giuliano $A R$, et al. Safety and reactogenicity of a quadrivalent human papillomavirus (types 6, 11, 16, 18) L1 viral-likeparticle vaccine in older adolescents and young adults. Hum Vaccin. 2011;7(7):768-775

31. Slade BA, Leidel L, Vellozzi C, et al. Postlicensure safety surveillance for quadrivalent human papillomavirus recombinant vaccine. JAMA. 2009;302 (7):750-757

32. Gee J, Naleway A, Shui I, et al. Monitoring the safety of quadrivalent human papillomavirus vaccine: findings from the Vaccine Safety Datalink. Vaccine. 2011;29(46):8279-8284

33. Chao C, Klein NP, Velicer CM, et al. Surveillance of autoimmune conditions following routine use of quadrivalent human papillomavirus vaccine. J Intern Med. 2012;271(2): 193-203

34. Klein NP, Hansen J, Chao C, et al. Safety of quadrivalent human papillomavirus vaccine administered routinely to females. Arch Pediatr Adolesc Med. 2012;166(12):1140-1148

35. Macartney KK, Chiu C, Georgousakis M, Brotherton JM. Safety of human papillomavirus vaccines: a review. Drug Saf. 2013;36(6):393-412

36. Arnheim-Dahlström L, Pasternak B, Svanström H, Sparén P, Hviid A. Autoimmune, neurological, and venous thromboembolic adverse events after immunisation of adolescent girls with quadrivalent human papillomavirus vaccine in Denmark and Sweden: cohort study. BMJ. 2013;347:55906

37. Grimaldi-Bensouda L, Guillemot D, Godeau B, et al; PGRx-AID Study Group. Autoimmune disorders and quadrivalent human papillomavirus vaccination of young female subjects J Intern Med. 2014;275(4):398-408

38. Scheller NM, Pasternak B, Svanström H, Hviid A. Quadrivalent human papillomavirus vaccine and the risk of venous thromboembolism. JAMA. 2014;312(2):187-188

39. Scheller NM, Svanström H, Pasternak B, et al. Quadrivalent HPV vaccination and risk of multiple sclerosis and other demyelinating diseases of the central nervous system. JAMA. 2015;313(1):54-61

40. Vichnin M, Bonanni P, Klein NP, et al. An overview of quadrivalent human papillomavirus vaccine safety: 2006 to 2015. Pediatr Infect Dis J. 2015;34(9):983-991

41. Dana A, Buchanan KM, Goss $M A$, et al. Pregnancy outcomes from the pregnancy registry of a human papillomavirus type 6/11/16/18 vaccine. Obstet Gynecol. 2009;114(6):1170-1178

42. Goss MA, Lievano F, Buchanan KM, Seminack MM, Cunningham ML, Dana A. Final report on exposure during pregnancy from a pregnancy registry for quadrivalent human papillomavirus vaccine. Vaccine. 2015;33(29):3422-3428 


\section{Safety Profile of the 9-Valent HPV Vaccine: A Combined Analysis of 7 Phase III}

Clinical Trials

Edson D. Moreira Jr, Stan L. Block, Daron Ferris, Anna R. Giuliano, Ole-Erik

Iversen, Elmar A. Joura, Pope Kosalaraksa, Andrea Schilling, Pierre Van Damme, Jacob Bornstein, F. Xavier Bosch, Sophie Pils, Jack Cuzick, Suzanne M. Garland, Warner Huh, Susanne K. Kjaer, Hong Qi, Donna Hyatt, Jason Martin, Erin Moeller,

Michael Ritter, Martine Baudin and Alain Luxembourg

Pediatrics; originally published online July 15, 2016; DOI: $10.1542 /$ peds. 2015-4387

\section{Updated Information \& Services}

Supplementary Material

References

Subspecialty Collections

Permissions \& Licensing

Reprints including high resolution figures, can be found at: /content/early/2016/07/14/peds.2015-4387.full.html

Supplementary material can be found at: /content/suppl/2016/07/14/peds.2015-4387.DCSupplemental. html

This article cites 40 articles, 4 of which can be accessed free at:

/content/early/2016/07/14/peds.2015-4387.full.html\#ref-list-1

This article, along with others on similar topics, appears in the following collection(s):

Infectious Disease

/cgi/collection/infectious_diseases_sub

Vaccine/Immunization

/cgi/collection/vaccine:immunization_sub

Information about reproducing this article in parts (figures, tables) or in its entirety can be found online at:

/site/misc/Permissions.xhtml

Information about ordering reprints can be found online: $/$ site/misc/reprints.xhtml

PEDIATRICS is the official journal of the American Academy of Pediatrics. A monthly publication, it has been published continuously since 1948. PEDIATRICS is owned, published, and trademarked by the American Academy of Pediatrics, 141 Northwest Point Boulevard, Elk Grove Village, Illinois, 60007. Copyright @ 2016 by the American Academy of Pediatrics. All rights reserved. Print ISSN: 0031-4005. Online ISSN: 1098-4275.

\section{American Academy of Pediatrics}




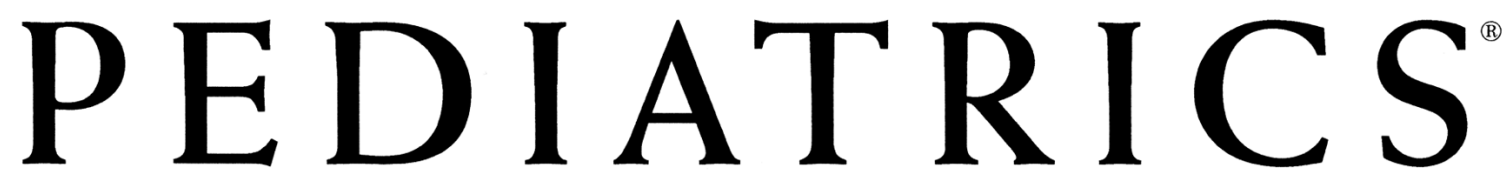

OFFICIAL JOURNAL OF THE AMERICAN ACADEMY OF PEDIATRICS

\section{Safety Profile of the 9-Valent HPV Vaccine: A Combined Analysis of 7 Phase III Clinical Trials}

Edson D. Moreira Jr, Stan L. Block, Daron Ferris, Anna R. Giuliano, Ole-Erik Iversen, Elmar A. Joura, Pope Kosalaraksa, Andrea Schilling, Pierre Van Damme, Jacob Bornstein, F. Xavier Bosch, Sophie Pils, Jack Cuzick, Suzanne M. Garland, Warner Huh, Susanne K. Kjaer, Hong Qi, Donna Hyatt, Jason Martin, Erin Moeller, Michael Ritter, Martine Baudin and Alain Luxembourg Pediatrics; originally published online July 15, 2016;

DOI: $10.1542 /$ peds.2015-4387

The online version of this article, along with updated information and services, is located on the World Wide Web at: /content/early/2016/07/14/peds.2015-4387.full.html

PEDIATRICS is the official journal of the American Academy of Pediatrics. A monthly publication, it has been published continuously since 1948. PEDIATRICS is owned, published, and trademarked by the American Academy of Pediatrics, 141 Northwest Point Boulevard, Elk Grove Village, Illinois, 60007. Copyright () 2016 by the American Academy of Pediatrics. All rights reserved. Print ISSN: 0031-4005. Online ISSN: 1098-4275.

\section{American Academy of Pediatrics}

